\title{
Diseño de un sistema inteligente y compacto de iluminación
}

\section{(Design of a Smart and Compact IIlumination System)}

\author{
Daniel López ${ }^{1}$, Daniel Mideros ${ }^{1}$
}

\begin{abstract}
Resumen:
La luz es un factor ambiental muy influyente en la calidad de vida y el rendimiento de las personas. Por esta razón es necesario crear e implementar tecnologías que ayuden a mantener ambientes sanos, en términos de iluminación, para los usuarios. Si a esto sumamos la gran cantidad de energía eléctrica que se desperdicia por malos hábitos de consumo y por el uso de dispositivos anticuados e ineficientes, resulta evidente la importancia del presente trabajo, el cual propone el diseño de una lámpara compacta y versátil que remplace fácilmente a los focos tradicionales con ventajas como un control automático para el encendido y apagado en función del movimiento registrado en la habitación, un control automático de nivel de iluminación para mantener niveles óptimos en todo momento y regulación del tono de la luz de acuerdo con la habitación y la hora del día. Para lograr lo anteriormente mencionado se utilizó tecnología LED, un microcontrolador y sensores que junto con técnicas de control gobiernan el comportamiento de la lámpara. Se establecieron varios parámetros de funcionamiento como nivel de iluminación y tono de la luz emitida los cuales se cumplieron dentro de ciertas condiciones operativas.
\end{abstract}

Palabras clave: luz; automatización; eficiencia energética; led.

\begin{abstract}
:
Light is a very influential environmental factor in the quality of life and the performance of people. For this reason, it is necessary to create and implement technologies that help maintain healthy environments, in terms of lighting, for users. If we add to this the large amount of electrical energy that is wasted due to bad consumption habits and the use of outdated and inefficient devices, the importance of this work is evident, which proposes the design of a compact and versatile lamp that easily replaces traditional light bulbs with advantages such as an automatic on and off control depending on the movement registered in the room, an automatic control of lighting level to maintain optimal levels at all times and regulation of the light tone according to the room and at the time of day. To achieve the aforementioned, LED technology, a microcontroller and sensors were used, which together with control techniques govern the behavior of the lamp. Several operating parameters were established such as lighting level and tone of the emitted light which were fulfilled within certain operating conditions.
\end{abstract}

Keywords: light; automation; energy efficiency; led.

\section{Introducción}

La iluminación es un factor primordial en la vida diaria de los humanos, puede afectar directamente a la salud y al rendimiento de las personas. Por esta razón es importante contar con una iluminación adecuada dentro de cada ambiente. Existen varios factores que llevan a la creación de un ambiente sano en términos de iluminación, estos son: la intensidad luminosa, el color, espectro de la luz y el ciclo de iluminación durante el día.

Una de las principales fuentes de consumo energético en los hogares es la iluminación, si consideramos que todavía se utilizan tecnologías anticuadas e ineficientes

\footnotetext{
${ }^{1}$ Universidad Tecnológica Equinoccial, Quito, Ecuador ( \{lfdf86770, dmideros\} @ute.edu.ec).
} 
y que dependen de los hábitos de consumo de las personas podemos ver como existen grandes posibilidades de mejora en este campo.

En la actualidad con la implementación de nuevas tecnologías se han desarrollado una amplia variedad de "focos inteligentes" que están disponibles en el mercado. Muchos de los cuales comparten características comunes y utilizan tecnologías similares. Entre las características principales que ofrecen estos dispositivos tenemos el control de nivel y de tono mediante aplicaciones especiales para teléfonos inteligentes, la detección de presencia para controlar el encendido y apagado y la posibilidad de programar horarios de funcionamiento. Sin embargo, el nivel de automatización es bastante bajo en la mayoría de modelos. Los fabricantes más relevantes en el momento son: Belkin, Stack y Sengled entre otros.

La implementación de un sistema de control de iluminación puede representar un ahorro energético de hasta un 35\% de acuerdo con la Agencia Internacional de energía (2015), estas cifras han llevado a un incremento en el uso de sistemas avanzados de control de iluminación, principalmente en los sectores comercial y público en los cuales la iluminación representa una gran parte del consumo eléctrico. Los sistemas de control de iluminación se clasifican en dos grupos: control On/Off y control de nivel.

El primero utiliza detectores de ocupación o temporizadores para apagar las luces cuando no son necesarias. Este tipo de sensores pueden ser montados en las paredes o techos de acuerdo con la habitación. El segundo utiliza foto detectores y un regulador de intensidad de manera que el nivel de iluminación se ajuste de acuerdo con el nivel de luz natural que exista, manteniendo un nivel predeterminado. En comparación con el sistema de control On/Off estos ofrecen una mayor posibilidad de ahorro y una mejor adaptación con las necesidades de las personas. (Yoshihisa, 2009)

Con estos antecedentes se propuso el diseño de una lámpara Led que mediante sensores integrados y un sistema de control, responda de manera inteligente a los cambios en su entorno al comprobar el nivel de iluminación, el tono de la luz y su encendido y apagado. La ventaja principal de esta lámpara es el hecho que tiene los dos tipos de sistemas de control y el elemento emisor de luz contenidos dentro de un mismo dispositivo, lo cual facilita su implementación.

\section{Metodología}

La metodología implementada para el desarrollo del prototipo fue el llamado Modelo $V$ que se describe en la Figura 1 (Ingenieure, 2004). En esta se pueden ver los pasos a seguir para obtener un producto a partir de una lista de requerimientos, como el diseño del sistema, el modelamiento y análisis y la integración del sistema.

La estructura básica de un sistema mecatrónico propuesta por Verein Deutscher Ingenieure (2004) consta de: fuente, actuadores, sistema base, sensores, procesamiento de la información, ambiente y usuario. Al adaptar estos elementos al proyecto obtenemos como resultado la Figura 2 que indica el diseño general del prototipo de lámpara inteligente. En esta podemos ver como la fuente de $9 \mathrm{~V}$ potencia los LEDs, los sensores detectan cambios en el entorno y los envían al sistema de control, el usuario, mediante los selectores establece los parámetros funcionales de la lámpara. Todos los elementos están interconectados con el sistema eléctrico y soportados por la carcasa.

\subsection{Sistema eléctrico}

El diseño del sistema eléctrico consta de tres partes fundamentales, la primera está formada por el circuito de iluminación es decir el arreglo de LEDs, las resistencias y los transistores necesarios para la operación del circuito. La segunda parte consta de un circuito lógico de control que interconecta los componentes electrónicos y permite el control de todo el sistema. Finalmente, la tercera parte está formada por la fuente de 
potencia que otorga el voltaje y la corriente necesarios para la operación de todos los componentes.

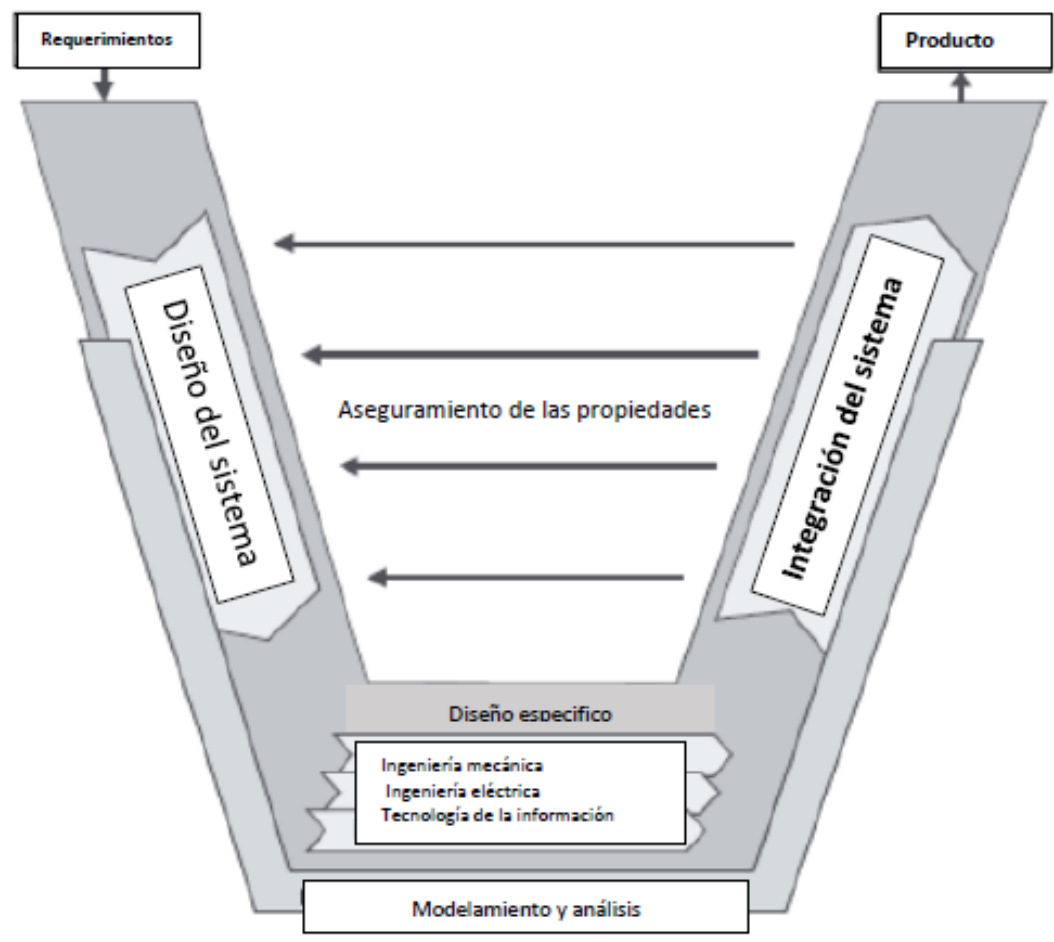

Figura 1. Modelo $V$ para el desarrollo de sistemas mecatrónicos Verein Deutscher Ingenieure (2004)

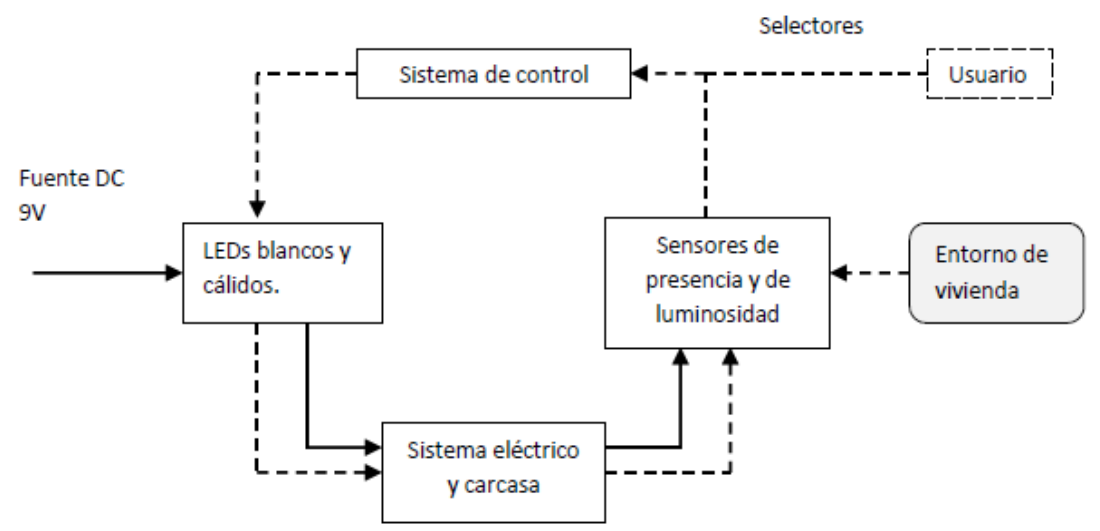

Figura 2. Estructura básica del prototipo de lámpara inteligente

El arreglo de leds consta de 24 LEDs SMD 5050 y 12 leds de $5 \mathrm{~mm}$. Los 24 LEDs SMD 5050 son los encargados de generar la totalidad del flujo luminoso es decir los $600 \mathrm{~lm}$, mientras que los LEDs de $5 \mathrm{~mm}$ son los encargados de generar el tono cálido de la luz. La Figura 3 muestra la placa electrónica en la cual se montarán todos los componentes. De acuerdo con el diseño realizado, el circuito de iluminación tiene una corriente de consumo máximo de $260 \mathrm{~mA}$. 


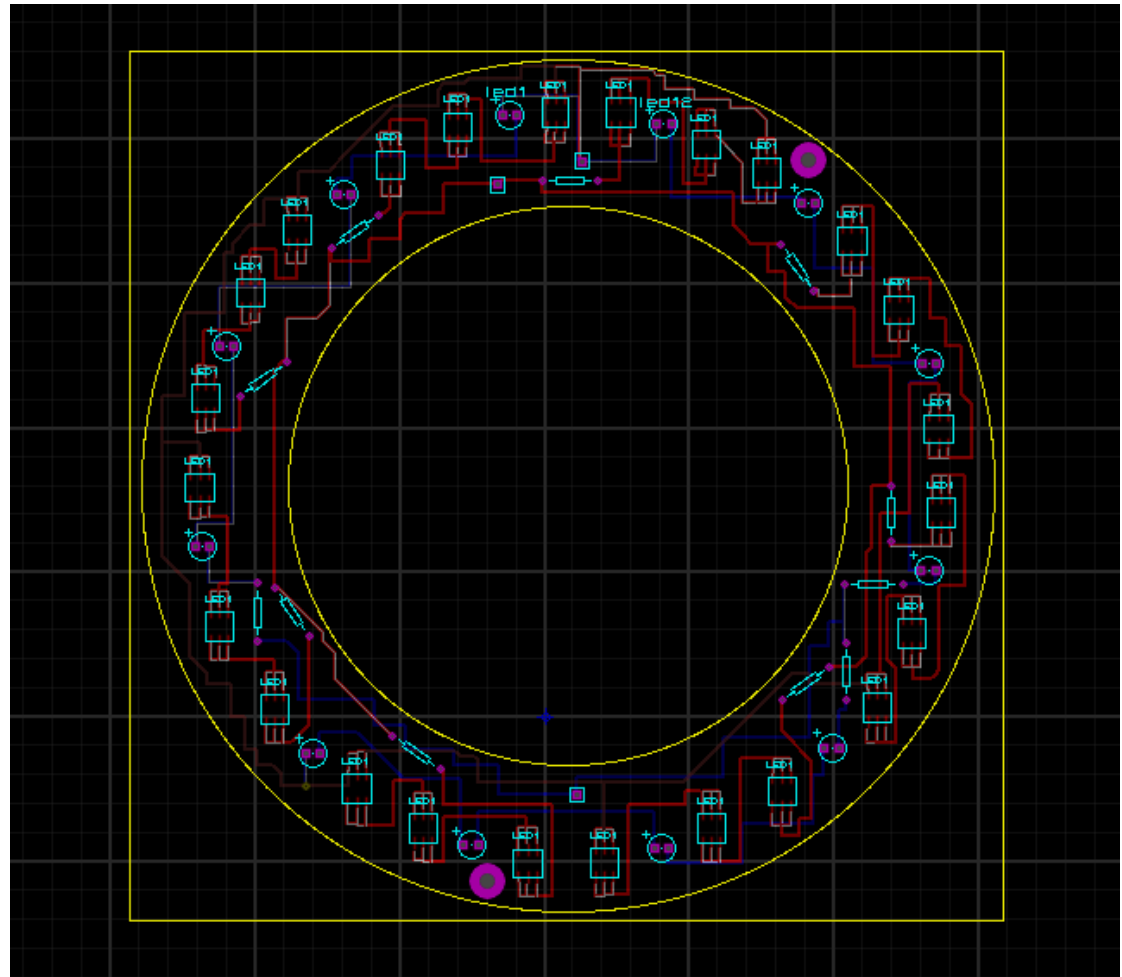

Figura 3. Diseño de la placa electrónica para el circuito de iluminación

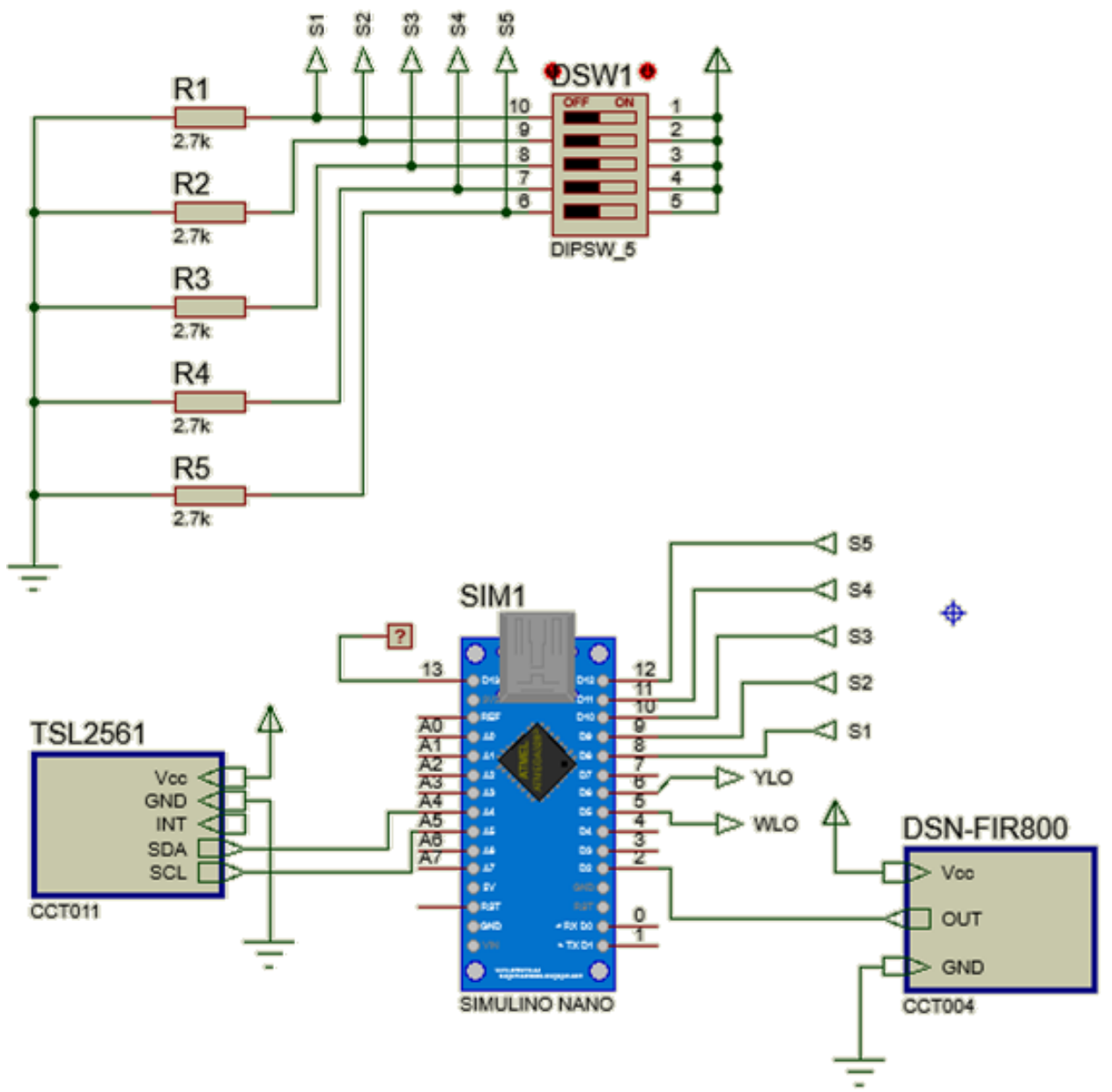

Figura 4. Diagrama de conexión del circuito lógico de control 
El circuito lógico de control tiene como centro un microcontrolador ATmega 328 representado en forma de un Arduino Nano, además consta de dos sensores (DSNFIR800 y TSL2561) y un selector para el ajuste de los parámetros de operación. El circuito de control posee una corriente máxima de consumo de $155 \mathrm{~mA}$. Ya que el diseño físico requiere que los diferentes elementos del circuito lógico se encuentren en lugares distintos dentro del prototipo no se los puede incluir a todos en una sola placa electrónica. La Figura 4 muestra el diagrama de conexión de este circuito.

La fuente de poder mostrada en la Figura 5, tiene como función principal la conversión del voltaje de 110VAC a 9VDC, esto se logró mediante la aplicación de una fuente capacitiva sin transformador con un rectificador tipo puente (Reston, 2004). Los datos iniciales para el diseño fueron: una corriente de salida de $450 \mathrm{~mA}$, un voltaje de salida de 9V, el voltaje de entrada que es de 110VAC y la frecuencia de $60 \mathrm{~Hz}$.

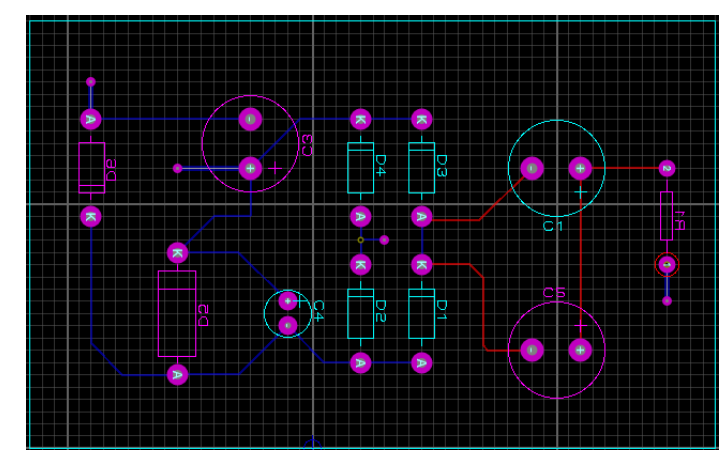

Figura 5. Diseño circuito para fuente de poder

\subsection{Carcasa y luminaria}

Para el diseño externo de la lámpara se tomaron como referencias productos existentes (Stack Labs Inc., 2015) y se llegó a la forma de disco convexo que sirvió como base para generar el diseño, la cubierta y luminaria. Como punto de partida se tomó a la cubierta por ser la pieza más grande del prototipo y a la cual van sujetos la mayor parte de los componentes. Se estableció un diámetro de $180 \mathrm{~mm}$, el cual da el espacio suficiente para alojar los componentes, manteniendo un diseño compacto.

La luminaria protege a los componentes internos y permite el paso de la luz generada por el arreglo de LEDs, por esta razón es necesario que el material sea traslucido. Adicionalmente a estos componentes se fabricó un contenedor que aísle los sensores de movimiento y de luminosidad de las posibles interferencias generadas por el cuerpo emisor. El contenedor de los sensores, al igual que la cubierta fueron impresos en 3D con ABS como material. Las diferentes piezas fueron modeladas de manera digital y se las puede observar en un ensamblaje parcial en la Figura 6.
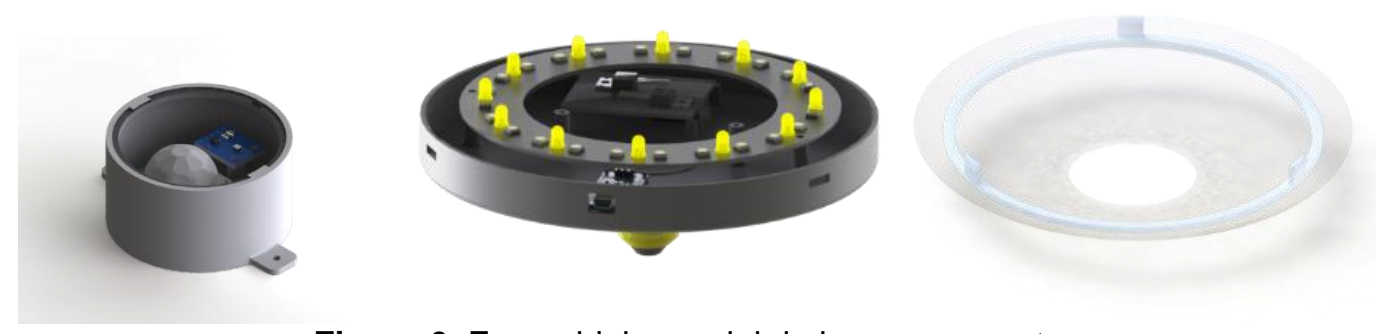

Figura 6. Ensamblaje parcial de los componentes 


\subsection{Sistema de control}

El algoritmo de control que gobierna el sistema sigue el diagrama de flujo de la Figura 7, el cual empieza por tomar los valores óptimos de iluminación y tono, los cuales se pueden observar en la Tabla 1. Luego realiza la calibración del sensor de movimiento e ingresa a un bucle en el cual se verifica constantemente el sensor de movimiento; en el caso que este detecte movimiento se realiza una lectura del sensor de iluminación para determinar si hace falta encender la lámpara y en qué modo. Si se necesita encender la lámpara se regula la intensidad lumínica mediante un controlador PID.

Tabla 1. Valores óptimos de iluminación y tono

\begin{tabular}{c|cc|c|c|c}
\hline Habitación & $\begin{array}{c}\text { Numero en } \\
\text { selector }\end{array}$ & $\begin{array}{c}\text { Nivel } \\
\text { mínimo (Ix) }\end{array}$ & $\begin{array}{c}\text { Nivel } \\
\text { óptimo (Ix) }\end{array}$ & $\begin{array}{c}\text { Tono } \\
\text { diurno }\end{array}$ & $\begin{array}{c}\text { Tono } \\
\text { nocturno }\end{array}$ \\
\hline $\begin{array}{c}\text { Dormitorios } \\
\text { Cuartos de } \\
\text { aseo }\end{array}$ & 1 & 100 & 150 & Blanco & Cálido \\
\hline $\begin{array}{c}\text { Cuartos de } \\
\text { estar }\end{array}$ & 2 & 100 & 150 & Blanco & Cálido \\
\hline $\begin{array}{c}\text { Cocinas } \\
\text { Cuartos de }\end{array}$ & 4 & 200 & 300 & Cálido & Cálido \\
\hline \begin{tabular}{c} 
trabajo/estudio \\
\hline
\end{tabular} & 5 & 100 & 150 & Blanco & Cálido \\
\hline
\end{tabular}

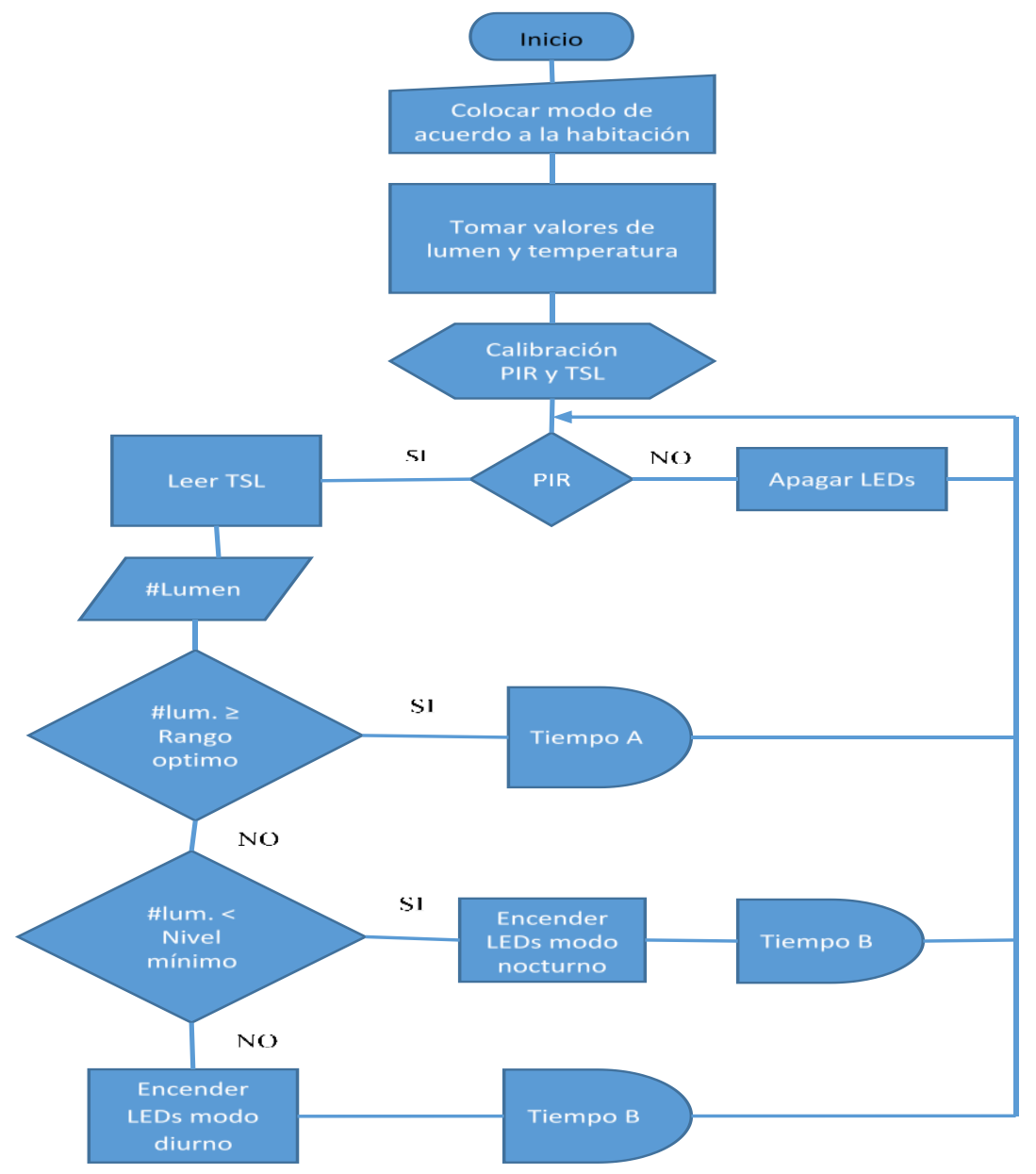

Figura 7. Diagrama de flujo del algoritmo de control 
El controlador PID controla la intensidad del flujo luminoso en función del nivel de luz medido por el sensor de luminosidad de modo que se obtenga un nivel de luminosidad estable, a pesar de las variaciones que pueden existir en el ambiente. Se utilizó el método de sintonización manual ("Sintonización de controladores por método heurístico," n.d.) bajo el cual se obtuvieron valores de $\mathrm{Kp}=1, \mathrm{Ki}=0.0001$ y $\mathrm{Kd}=0.0001$ con los cuales el sistema alcanza el valor de referencia de manera rápida y sin un error significativo, como se puede observar en la Figura 8, el tiempo de estabilización del sistema es de 200 mili segundos aproximadamente.

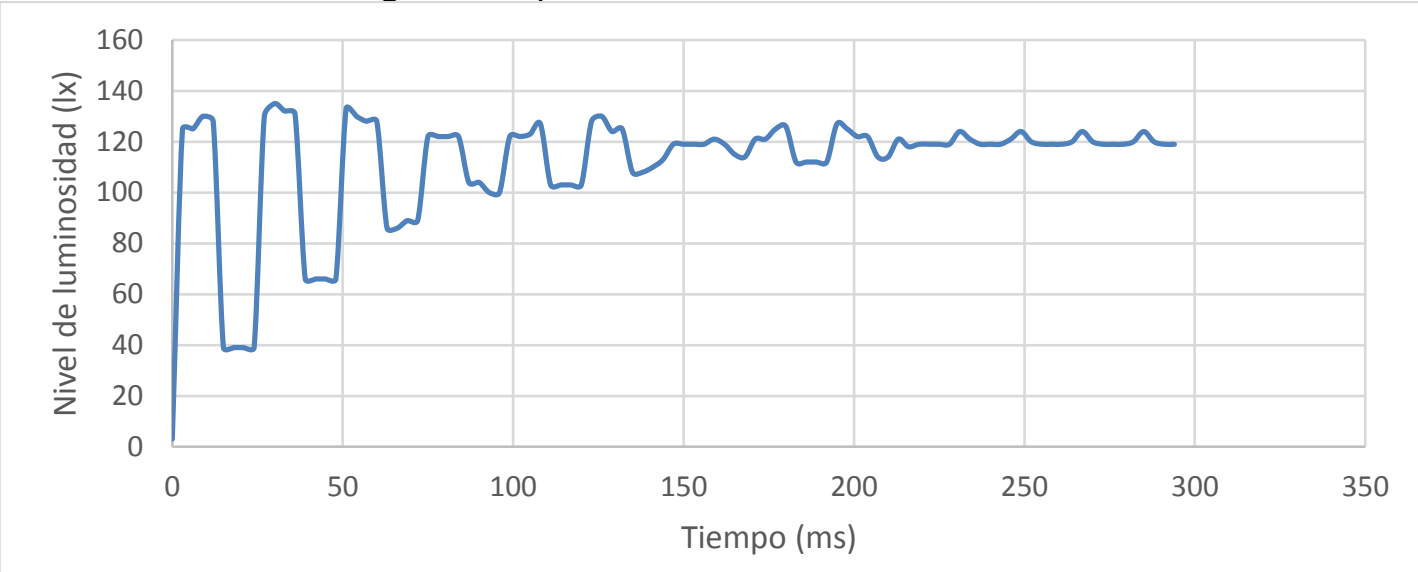

Figura 8. Tiempo de respuesta del controlador PID

\section{Resultados y discusión}

La toma de datos de iluminación se realizó en un ambiente controlado, pero no aislado de la iluminación natural, se tomaron medidas a diferentes horas del día y bajo diferentes condiciones climáticas para obtener datos válidos.

\section{1. Área optima de iluminación}

Para el cálculo del área óptima de iluminación se utilizó el método de la cavidad zonal (Laszlo, 2010). Basado en las tablas propuestas por el manual de luminotecnia de Laszlo (2010) se obtuvo un coeficiente de iluminación (cu) en el rango de 0.38 a $0.48 \mathrm{si}$ se toma en cuenta una reflectancia de pared y techo igual al $70 \%$, la cual depende del acabado superficial que posean las mismas. Entonces se puede aplicar la ecuación (1) despejando la variable de superficie, para obtener un rango del área óptima de iluminación. En la Tabla 2 se pueden observar los datos necesarios para el cálculo del área óptima de iluminación y como esta depende de la altura $h$, para el cálculo se han tomado valores entre 1.2 y 1.7 metros pues es el rango promedio de altura del plano de trabajo de una habitación estándar.

$$
S=\frac{\varphi t \cdot c u \cdot f m}{E m}
$$

Tabla 2. Rango de superficies optimas de iluminación.

\begin{tabular}{rl|l|lll}
\hline $\boldsymbol{\phi t}(\mathbf{I m})$ & $\mathbf{f m}$ & $\mathbf{E m}(\mathbf{l u x})$ & $\mathbf{h}(\mathbf{m})$ & $\mathbf{c u}$ & $\mathbf{S}\left(\mathbf{m}^{2}\right)$ \\
\hline 907.2 & 1 & 150 & 1.2 & 0.38 & $\mathbf{2 . 3 0}$ \\
\hline & & & 1.3 & 0.4 & $\mathbf{2 . 4 2}$ \\
& & & 1.4 & 0.42 & $\mathbf{2 . 5 4}$ \\
\hline & & 1.5 & 0.44 & $\mathbf{2 . 6 6}$ \\
\hline & & & 1.6 & 0.46 & $\mathbf{2 . 7 8}$ \\
& & & 1.7 & 0.48 & $\mathbf{2 . 9 0}$
\end{tabular}


Donde:

$\varphi t=$ flujo luminoso producido por el cuerpo emisor expresado en lúmenes

$\mathrm{fm}=$ factor de mantenimiento, en este caso es igual a 1 ya que se trata de un dispositivo completamente nuevo

$E m$ = es el nivel medio de iluminación sobre un plano de trabajo expresado en luxes

$\mathrm{h}=$ es la distancia que existe entre la luminaria y el plano de trabajo en metros

$c u=$ es el coeficiente de iluminación

$S=$ es la superficie óptima de iluminación en metros cuadrados

\subsection{Resultados de iluminación}

Las medidas de iluminación fueron tomadas en dos circunstancias diferentes: un día soleado y un día nublado, en cinco habitaciones distintas que corresponden a los distintos modos de funcionamiento de la lámpara y a diferentes horas del día. En la Tabla 3 se pueden apreciar, los datos obtenidos de la medición del nivel de iluminación en cada una de las habitaciones. Se encuentran resaltados los valores correspondientes a los períodos en los cuales la lámpara se encuentra encendida. Todos los valores se miden en luxes.

Tabla 3. Resultados de iluminación de acuerdo con la habitación

\begin{tabular}{|r|r|r|r|r|r|r|r|r|r|r|r|}
\hline & \multicolumn{3}{|c|}{ dormitorio } & \multicolumn{3}{c|}{ baño } & \multicolumn{3}{c|}{ sala } & \multicolumn{3}{c|}{ cocina } & \multicolumn{2}{c|}{ estudio } \\
\hline hora: & Soleado & Nublado & Soleado & Nublado & Soleado & Nublado & Soleado & Nublado & Soleado & Nublado \\
\hline $5: 00$ & 152 & 152 & 162 & 146 & 254 & 253 & 145 & 146 & 321 & 332 \\
\hline $7: 00$ & 174 & 123 & 161 & 151 & 247 & 248 & 122 & 152 & 353 & 329 \\
\hline $9: 00$ & 216 & 186 & 185 & 117 & 220 & 251 & 221 & 185 & 540 & 421 \\
\hline $11: 00$ & 252 & 205 & 204 & 124 & 251 & 225 & 329 & 235 & 626 & 485 \\
\hline $14: 00$ & 354 & 290 & 174 & 109 & 293 & 247 & 261 & 193 & 432 & 319 \\
\hline $17: 00$ & 146 & 152 & 192 & 120 & 324 & 281 & 153 & 152 & 331 & 324 \\
\hline $19: 00$ & 151 & 154 & 154 & 148 & 253 & 246 & 146 & 150 & 315 & 326 \\
\hline $21: 00$ & 153 & 151 & 167 & 154 & 254 & 252 & 152 & 148 & 319 & 322 \\
\hline
\end{tabular}

En todos los casos existen horas del día en los cuales la lámpara no se enciende dado que los niveles de luminosidad natural superan los niveles mínimos sugeridos mostrados en la Tabla 1.

\subsection{Luminancia y calidad del haz de luz}

Para determinar la calidad de haz de luz producida por la lámpara se tomaron tres medidas en distintos puntos dentro del área óptima calculada como se muestra en la Figura 9. El primer punto se encuentra directamente debajo de la luminaria, el punto dos está a 0.38 metros del primero y el tercer punto se encuentra en el borde del área óptima es decir a 0.75 metros de $\mathrm{P} 1$. Todos los puntos se encuentran sobre el plano de trabajo situado a 1.2 metros de la luminaria.

Los resultados se muestran en la Figura 10 donde se puede apreciar un decaimiento considerable a medida que el punto de medición se aleja del centro del área óptima, es decir deja de ser perpendicular a la luminaria, esto demuestra que el haz de luz tiene un ángulo reducido que afecta a la luminancia percibida en el ambiente. A una distancia superior a los 0.5 metros la luminancia cae por debajo del nivel mínimo establecido de $120 \mathrm{~lx}$. 


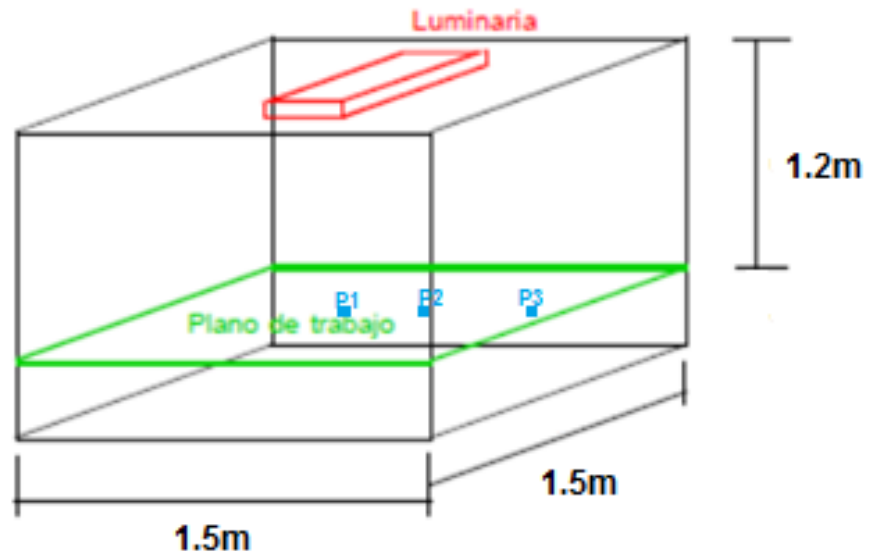

Figura 9. Puntos de medición de luminancia

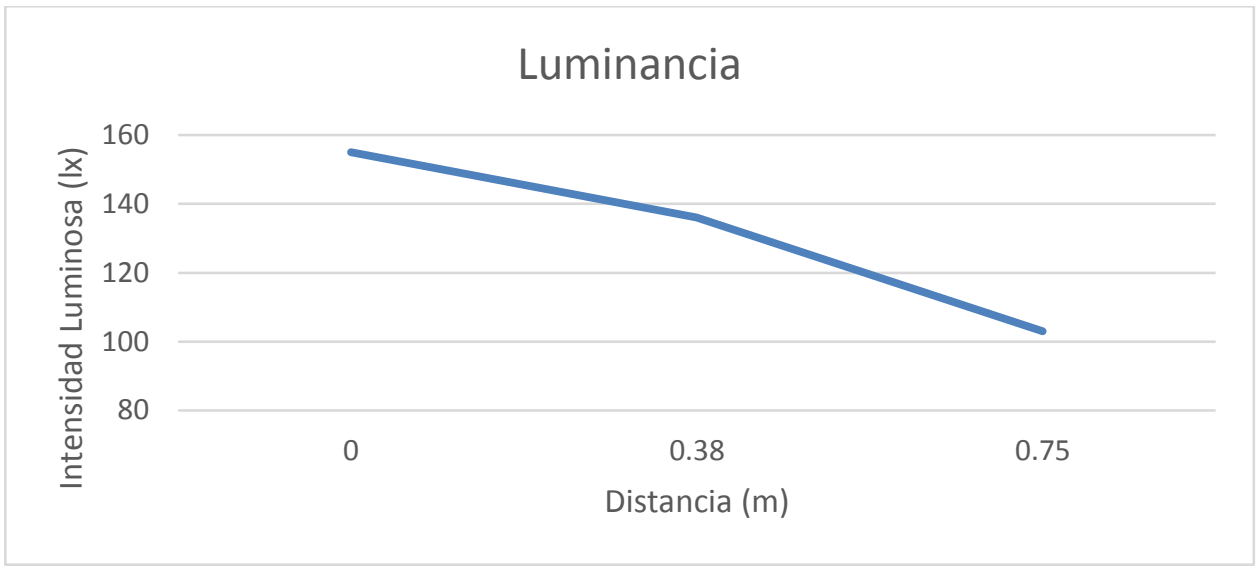

Figura 10. Decaimiento de la intensidad luminosa en función de la distancia

\section{Conclusiones y recomendaciones}

Se determinó que la intensidad luminosa y el tono de la luz son los factores físicos más importantes al momento de ofrecer un sistema de iluminación ergonómico, por esta razón se diseñó el prototipo con cinco modos de fruncimiento: dormitorio, baño, sala de estar, cocina y estudio.

Se creó una lámpara con un diseño compacto y de fácil implementación que remplazaría fácilmente a los focos tradicionales.

Se generó un algoritmo de control que gobierna el funcionamiento automático de la lámpara con ayuda de un controlador PID.

El componente que presentó la mayor dificultad para su fabricación fue el arreglo de LEDs pues la tecnología disponible en el país es limitada. Si bien se alcanzó el flujo luminoso de $800 \mathrm{~lm}$ propuesto, el haz de luz pierde intensidad con la distancia y no se genera una iluminación uniforme en el plano de trabajo.

Las pruebas demostraron que la lámpara mantiene los niveles óptimos en muchos casos a pesar de estar instalada en habitaciones con dimensiones superiores a el área óptima calculada.

A futuro se podrían buscar alternativas para la ubicación del sensor de luminosidad u otros métodos de compensación que ayuden a eliminar los errores de lectura de los niveles de intensidad luminosa que existen en el ambiente.

Otro tema que se podría revisar es la posibilidad de implementar tecnologías de comunicación inalámbrica como bluetooth o wi-fi que, en conjunto con una aplicación 
móvil o web, permitan el control manual de los parámetros de la lámpara inteligente, así como la posibilidad de programar horarios y alarmas en el dispositivo.

Finalmente sería recomendable investigar sobre la posibilidad de implementar algoritmos de aprendizaje de modo que el dispositivo pueda adaptarse de mejor manera a su entorno y al usuario en particular.

\section{Bibliografía}

Baggini, A., \& Sumper, A. (2012). Electrical Energy Efficiency: Technologies and Applications. Hoboken, NJ, USA: John Wiley \& Sons. Recuperado de http://site.ebrary.com/lib/alltitles/docDetail.action?doclD=10542505

Conejo, A. J., Arroyo, J. M., \& Milano, F. (2007). Instalaciones eléctricas. España: McGraw-Hill España. Recuperado de http://site.ebrary.com/lib/alltitles /docDetail.action?doc/D=10491395

Dunning, S., \& Thuman, A. (2012). Efficient Lighting Applications and Case Studies. Lilburn, GA, USA: The Fairmont Press, Inc. Recuperado de http://site.ebrary.com/lib/alltitles/docDetail.action?doclD=10849093

EcoHabitar. (n.d.). La iluminación biológica crea ambientes sanos y optimistas. Retrieved from http://www.ecohabitar.org/la-iluminacion-biologicacrea- ambientes-sanos-yoptimistas/

Fernández, R. (2013). Análisis y Diseño de Sistemas de Control Digital. México, D.F.: McGraw-Hill/ Interamericana Editores.

Ingenieure, V. D. (2004, June). Design methodology for mechatronic systems. Düsseldorf, Germany.

Laszlo, C. (2010). Manual de luminotecnia para interiores. Manual de luminotecnia para interiores. Buenos Aires, Argentina

López, D., Mideros, D. \& Moya, M. (2017) Diseño e Implementación de un Prototipo de Lámpara Inteligente. INCISCOS 2017. Quito, Ecuador. Pronto a publicarse.

Reston, C. (2004). Transformerless Power Supplies: Resistive and Capacitive. USA.

Sánchez Fleitas, N., Comas Rdoríguez, R., García Lorenzo, M., \& Riverol Quesada, A. (2016). Modelo de manejo de datos, con el uso de inteligencia artificial, para un sistema de información geográfica en el sector energético. Enfoque UTE, 7(3), pp. 95 - 109. https://doi.org/https://doi.org/10.29019/enfoqueute.v7n3.108

Sintonización de controladores por método heurístico: "prueba y error." (n.d.). Retrieved October 29, 2015, from

http://www.instrumentacionycontrol.net/cursos-libres/automatizacion/cursosintonizacion controladores/item/394-sintonizaci\%C3\%B3n-decontroladores-por-m\%C3\%A9todoheur\%C3\%ADstico-prueba-y-error.html

Yoshihisa, T. (2009). Energy saving liehting efficiency technologies. Quarterly Review, 5971. 